\title{
Multiple shocks around the low-luminosity protostar IRAS 16293-2422*
}

\author{
A. Castets ${ }^{1}$, C. Ceccarelli ${ }^{1,2}$, L. Loinard ${ }^{3}$, E. Caux ${ }^{4}$, and B. Lefloch ${ }^{2}$ \\ 1 Observatoire de Bordeaux, BP 89, 33270 Floirac, France \\ 2 Laboratoire d'Astrophysique, Observatoire de Grenoble, BP 53, 38041 Grenoble Cedex 09, France \\ 3 Instituto de Astronomía, UNAM, Apdo Postal 72-3 (Xangari), 58089 Morelia, Michoacán, México \\ 4 CESR CNRS-UPS, BP 4346, 31028 Toulouse Cedex 04, France
}

Received 1 February 2001 / Accepted 2 May 2001

\begin{abstract}
Using the IRAM $30 \mathrm{~m}$ and SEST $15 \mathrm{~m}$ telescopes we mapped an area covering $300^{\prime \prime} \times 220^{\prime \prime}$ around IRAS 16293-2422 in the $\mathrm{N}_{2} \mathrm{H}^{+} J=1 \rightarrow 0, \mathrm{H}_{2} \mathrm{CO} J=3_{1,3} \rightarrow 2_{1,2}, \mathrm{SiO} J=2 \rightarrow 1 J=3 \rightarrow 2$ and $J=5 \rightarrow 4$ molecular transitions. Five positions were also observed in the FIR, between $45 \mu \mathrm{m}$ and $200 \mu \mathrm{m}$, with the Long Wavelength Spectrometer on board ISO, revealing only [C II] $158 \mu \mathrm{m}$ and [O I] $63 \mu \mathrm{m}$ emission. All these observations are used to reconstruct the complex morphology of the region, in which several outflows from several sources co-exist. The $\mathrm{N}_{2} \mathrm{H}^{+}$line emission is strong and centered on $16293 \mathrm{E}$, a recently discovered low mass and very young protostar situated South-East of IRAS 16293-2422. Only weaker $\mathrm{N}_{2} \mathrm{H}^{+}$line emission is associated with the binary system IRAS 16293-2422, presumably because the gas surrounding it is warmer. In addition to the previously known North-East-South-West outflow powered by IRAS 16293-2422, we suggest the existence of a second outflow in this binary system. We also report the discovery of a North-West-South-East flow driven by $16293 \mathrm{E}$. The impact of the outflows with the ambient cloud is probed by the $\mathrm{SiO}$ and $\mathrm{H}_{2} \mathrm{CO}$ maps, which reveal the presence of at least four, possibly six shocked regions. We discuss in some detail the nature of the shocks, deriving densities, temperatures and column densities of the shocked gas of the various observed components. We suggest that the wind and ambient shocks are probed by the high and low velocity components observed in the $\mathrm{SiO}$ lines. The morphology of the $\mathrm{H}_{2} \mathrm{CO}, \mathrm{SiO}$ and [O I] line emissions are rather different, showing almost all kind of combinations. We discuss the origin of these differences in terms of the age of the shocks, the pre-shock densities and of the composition of the ices which are partially desorbed by the shocks.
\end{abstract}

Key words. ISM: abundances - ISM: individual: IRAS 16293-2422 - ISM: molecules - stars: formation

\section{Introduction}

The molecular outflows powered by low-luminosity protostars are known to exhibit molecular abundances very different from those of the quiescent interstellar medium. Indeed, processes such as endothermic reactions, sublimation of ices, and disruption of dust grain cores lead to a "shock chemistry" which is totally different from that at work in the quiescent interstellar medium (e.g. van Dishoeck \& Blake 1998). For example, molecules such

Send offprint requests to: A. Castets,

e-mail: Alain.castets@observ.u-bordeaux.fr

* Based on observations collected at IRAM, Spain, at the European Southern Observatory Chile, and on observations with ISO, an ESA project with instruments funded by ESA Member States (especially the PI countries: France, Germany, The Netherlands and the UK) with the participation of ISAS and NASA. as $\mathrm{SiO}$ and $\mathrm{CH}_{3} \mathrm{OH}$, are found to be up to a million times more abundant in outflows than in quiescent molecular clouds (Bachiller \& Perez Gutierrez 1997). The generally accepted view is that the shocks created by the supersonic flows emanating from protostars lead to the desorption of the dust grain mantles and possibly to the disruption of the grain itself, which injects "unusual" chemical species in the gas phase. Those species can then be observed directly, like $\mathrm{CH}_{3} \mathrm{OH}$ or $\mathrm{H}_{2} \mathrm{CO}$, or after some chemical processing, like $\mathrm{SiO}$, which seems to result from the oxidization of the $\mathrm{Si}$ atom released from the dust grains (Schilke et al. 1997). The processes responsible for the appearance of "unusual" chemical species can be very fast compared with the cooling time scale of the gas. Shock chemistry is therefore strongly time-dependent and the relative abundances of molecular species like $\mathrm{SiO}, \mathrm{CH}_{3} \mathrm{OH}, \mathrm{H}_{2} \mathrm{CO}$ or the sulfur-containing molecules (Bachiller \& Pérez-Gutiérez 1998) reflect the evolution states of the outflow and its 
powering source. Consequently, those tracers of shocked material are important tools for the study of starformation.

The low-luminosity Class 0 protostar IRAS 16293$2422\left(15 L_{\odot}\right)$ is located in the small L1689N molecular cloud in the $\rho$ Ophiuchus complex at a distance of 120 pc (Knude \& Hog 1998). It lies in a fairly complex environment, where several protostars and multiple outflows coexist (Walker et al. 1986). The envelope of IRAS 162932422 has been the target of numerous studies (Blake et al. 1994; van Dishoeck et al. 1995). The physical structure (density and temperatures profiles) of this envelope has been recently reconstructed by Ceccarelli et al. (2000a,b, 2001). The outflows powered by IRAS 16293-2422 have been traced in CO by Walker et al. (1986) among others, in [O I] by Ceccarelli et al. (1997) and more recently in $\mathrm{SiO}$ by Hirano et al. (2000). However, few studies have been devoted to the study of the contents and dynamics of the whole region. The disentanglement of the various components is difficult, but necessary, to understand the structure and the evolution of the region. The recent $J=2 \rightarrow 1 \mathrm{SiO}$ maps published by Hirano et al. (2000; hereafter HI2000) provided important informations on that structure, but the more recent identification of a new protostar in the vicinity of IRAS 16293-2422 by Loinard et al. (in preparation) suggests that they misinterpreted some of the structures. Here, we present new observations of several tracers of quiescent and shocked interstellar material, and use these observations to provide a complete description of the region surrounding IRAS 16293-2422. The observations are described in Sect. 2, the results are presented in Sect. 3, and discussed in Sect. 4. Section 5 contains the conclusions.

\section{Observations}

Using the IRAM and SEST millimeter telescopes, large scale maps of the region around IRAS 162932422 have been obtained in the $J=1 \rightarrow 0, J=3 \rightarrow 2$, and $J=5 \rightarrow 4$ transitions of $\mathrm{SiO}$, the $J=1 \rightarrow 0$ transition of $\mathrm{N}_{2} \mathrm{H}^{+}$, and the $J=3_{1,3} \rightarrow 2_{1,2}$ transition of $\mathrm{H}_{2} \mathrm{CO}$. Some specific positions inside those maps were also observed in the fine structure $63 \mu \mathrm{m}$ line of $\mathrm{O}$ with ISO.

The coordinates $(\Delta \alpha, \Delta \delta)$ of all the maps shown here are offsets relative to the position of the $16293 \mathrm{~B}$ component of the binary system IRAS $16293-2422$ at $\alpha(2000)=$ $16^{\mathrm{h}} 32^{\mathrm{m}} 22 \mathrm{~s} 6, \delta(2000)=-24^{\circ} 28^{\prime} 33^{\prime \prime}$ (the $16293 \mathrm{~A}$ component is located at $4^{\prime \prime}$ South and $2^{\prime \prime}$ East from the B component, Looney et al. 2000).

\subsection{Millimeter observations}

An area covering $300^{\prime \prime} \times 220^{\prime \prime}(\Delta \alpha \times \Delta \delta)$ was observed in the $J=1 \rightarrow 0$ transition of $\mathrm{N}_{2} \mathrm{H}^{+}$at $93.173 \mathrm{GHz}$, and in the $J=3_{1,3} \rightarrow 2_{1,2}$ transition of $\mathrm{H}_{2} \mathrm{CO}$ at 211.211 $\mathrm{GHz}$ with the SEST telescope in July 1998. In addition a $50^{\prime \prime} \times 60^{\prime \prime}$ higher resolution map centered on IRAS $162933-2422$, of the $\mathrm{H}_{2} \mathrm{CO} J=3_{1,3} \rightarrow 2_{1,2}$ tran- sition, was obtained with the $30-$ meter IRAM telescope in March 2000. A $200^{\prime \prime} \times 150^{\prime \prime}$ area was mapped in the $J=2 \rightarrow 1, J=3 \rightarrow 2$, and $J=5 \rightarrow 4$ transitions of $\mathrm{SiO}$ at $86.846,130.268 \mathrm{GHz}$ and $217.104 \mathrm{GHz}$ respectively. The portions of these $\mathrm{SiO}$ maps, corresponding to $\Delta \delta \leq$ 0 , were obtained with the 15 -meter SEST telescope in January 1997 and July 1998, while the portions corresponding to $\Delta \delta \geq 0$ were obtained with the IRAM 30meter telescope in June 1997 and April 1998.

All data were obtained in position switching mode, with the OFF position located at $\Delta \alpha=-180^{\prime \prime}, \Delta \delta=0^{\prime \prime}$ from the center of IRAS 16293-2422. All maps were obtained on regular grids, with spacings of $12^{\prime \prime}$, and $24^{\prime \prime}$ at IRAM and SEST, respectively. Even though the $1 \mathrm{~mm}$ observations are not Nyquist sampled, we smoothed our $1 \mathrm{~mm}$ data to the $3 \mathrm{~mm}$ data beamsizes. In theory, full beam spacing does not allow proper smoothing of the data. However, except in pathological source morphologies, a full beam spacing gives reasonably good results (the angular resolutions corresponding to these observations are listed in Table 1). Both at IRAM and SEST, the pointing and focus drifts were monitored using planets or strong extragalactic continuum sources. The pointing corrections were found to be always below $3^{\prime \prime}$ and $5^{\prime \prime}$ at IRAM and SEST respectively. All intensities are expressed in units of main beam brightness temperature. The main beam efficiencies at each frequency are listed in Table 1. The relative intensity scales of the SEST and IRAM telescopes were carefully checked by comparing $\mathrm{SiO}$ spectra smoothed to a common angular resolution. They were found to be consistent within $15 \%$.

IRAM observations: The Northern $(\Delta \delta \geq 0)$ parts of the three $\mathrm{SiO}$ maps and the $\mathrm{H}_{2} \mathrm{CO}$ map were obtained with the IRAM 30-meter telescope, located at an altitude of 2920 meters near the summit of Pico Veleta in Southern Spain. The 3, 2, and $1.3 \mathrm{~mm}$ SIS receivers available at the 30 -meter were used together to map simultaneously all three transitions of $\mathrm{SiO}$. The $\mathrm{H}_{2} \mathrm{CO} J=3_{1,3} \rightarrow 2_{1,2}$ transition was observed simultaneously with other molecular transitions not presented here. The image sideband rejections of all receivers were always higher than $10 \mathrm{~dB}$. The three receivers were connected to units of the autocorrelator set to provide spectral resolutions of 40, 40, and $80 \mathrm{kHz}$ at 87,130 , and $217 \mathrm{GHz}$, respectively. At all frequencies, the velocity resolutions are of the order of $0.1-0.2 \mathrm{~km} \mathrm{~s}^{-1}$ (Table 1).

SEST observations: The SEST telescope is a 15-meter single dish millimeter telescope operated jointly by ESO and a consortium of Swedish institutions. It is located at an altitude of about 2300 meters in La Silla in Chile. To obtain all the maps shown here (Southern part of the $\mathrm{SiO}$ maps, $\mathrm{N}_{2} \mathrm{H}^{+}$, and $\mathrm{H}_{2} \mathrm{CO}$ maps), the two dual SIS receivers $(3 / 2 \mathrm{~mm}$, and $3 / 1.3 \mathrm{~mm})$ were used alternatively. They were always connected to the high resolution acoustooptical spectrometer available at the SEST, which provides a spectral resolution of $43 \mathrm{kHz}$. At the frequencies considered here, that corresponds to a velocity resolution 
Table 1. Summary of all millimetric observations with the appropriate observing parameters.

\begin{tabular}{lllcccc}
\hline Transition & $\begin{array}{c}\text { Frequency } \\
(\mathrm{GHz})\end{array}$ & Instrument & $\begin{array}{c}H P B W \\
\left({ }^{\prime \prime}\right)\end{array}$ & $\begin{array}{c}\eta_{\mathrm{mb}} \\
\begin{array}{c}\Delta \nu \\
(\mathrm{kHz})\end{array}\end{array}$ & $\begin{array}{c}\Delta v \\
\left(\mathrm{~km} \mathrm{~s}^{-1}\right)\end{array}$ \\
\hline $\mathrm{SiO}(2 \rightarrow 1)$ & \multirow{2}{*}{86.846} & IRAM & 27 & 0.76 & 40 & 0.14 \\
& & SEST & 57 & 0.77 & 43 & 0.15 \\
$\mathrm{SiO}(3 \rightarrow 2)$ & \multirow{2}{*}{130.268} & IRAM & 18 & 0.63 & 40 & 0.09 \\
& & SEST & 40 & 0.64 & 43 & 0.1 \\
$\mathrm{SiO}(5 \rightarrow 4)$ & 217.104 & IRAM & 12 & 0.45 & 80 & 0.11 \\
& & SEST & 25 & 0.47 & 43 & 0.06 \\
$\mathrm{~N}_{2} \mathrm{H}^{+}(1 \rightarrow 0)$ & 93.173 & SEST & 50 & 0.75 & 43 & 0.14 \\
$\mathrm{H}_{2} \mathrm{CO}\left(3_{1,3} \rightarrow 2,2\right)$ & 211.211 & SEST & 26 & 0.48 & 43 & 0.06 \\
& & IRAM & 12 & 0.45 & 40 & 0.06 \\
\hline
\end{tabular}

of $0.1-0.2 \mathrm{~km} \mathrm{~s}^{-1}$ (Table 1), comparable to that of the IRAM data.

\subsection{ISO observations}

The five positions reported in Table 2 were observed with the Long Wavelength Spectrometer (hereafter LWS: Clegg et al. 1996) in the AOTL01 (grating) mode, and the Short Wavelength Spectrometer (hereafter SWS: de Graauw et al. 1996) in the AOTS02 (grating) mode, both onboard the ISO satellite (Kessler et al. 1996). The positions of the ISO observations together with the ISO beam are reported in Fig. 4.

The LWS spectra cover the spectral range $45-200 \mu \mathrm{m}$ at a spectral resolution of about 250 (i.e. $\sim 1500 \mathrm{~km} \mathrm{~s}^{-1}$ ). The beam width of ISO at that wavelength is $\sim 80^{\prime \prime}$ (Swinyard et al. 1996). Three scans (for a total integration time of $925 \mathrm{~s}$ ) were obtained at each position. The data were reduced with the Off-Line-Package (OLP) version 7 and with the standard reduction package ISAP. Fringes with amplitude sometimes comparable to that of the continuum had to be removed. For such large amplitude, the removal process contributes significantly to the errors in the estimates of the line fluxes - particularly longwards of $\sim 100 \mu \mathrm{m}$.

The LWS spectra consist of a continuum on which spectral features are superimposed. To obtain the fluxes of the lines, the best first order polynomial fit to the continuum adjacent to each line was removed from the spectrum. The continuum is 2 to 50 times stronger than the lines, so the baseline removal is another important source of uncertainty in the reported line fluxes, much larger, indeed, than the statistical errors. The absolute flux calibration accuracy of LWS is better than $30 \%$ (Swinyard et al. 1996). Besides the fluxes at the five positions considered here, Table 2 reports the flux in the region surrounding the outflow (called OFF-OUTFLOW) reported by Caux et al. (1999), and obtained by averaging ten OFF positions.

The SWS spectra were used to search for the 0-0 S(0) and $0-0 \mathrm{~S}(1)$ lines of $\mathrm{H}_{2}$ at 28 and $17 \mu \mathrm{m}$ respectively, and the $\mathrm{Si}^{+}$line at $34 \mu \mathrm{m}$. About $1000 \mathrm{~s}$ of integration were obtained at each position. The spectral resolution of SWS for point sources is $\sim 2000$ (i.e. $\sim 200 \mathrm{~km} \mathrm{~s}^{-1}$ ) at all wave-
Table 2. Intensity of the $[\mathrm{O}$ I] line at $63 \mu \mathrm{m}$ in 5 positions around IRAS 16293-2422, and at the OFF position. The line flux of the OFF-OUTFLOW correspond to the average of ten OFF positions (Caux et al. 1999).

\begin{tabular}{lccc}
\hline Position Name & $\begin{array}{c}\Delta \alpha \\
\left({ }^{\prime \prime}\right)\end{array}$ & $\begin{array}{r}\Delta \delta \\
\left({ }^{\prime \prime}\right)\end{array}$ & $\begin{array}{c}\text { OI line intensity } \\
\left(10^{-12} \mathrm{erg} \mathrm{s}^{-1} \mathrm{~cm}^{-2}\right)\end{array}$ \\
\hline W1,W2 & -30 & +30 & $7.9 \pm 0.5$ \\
E1 & +20 & +30 & $4.7 \pm 0.4$ \\
SWB & -50 & -40 & $3.4 \pm 0.7$ \\
E2 & +80 & +60 & $3.3 \pm 0.4$ \\
EB & +80 & -10 & $3.5 \pm 0.4$ \\
OFF-OUTFLOW & & & $0.8 \pm 0.2$ \\
\hline
\end{tabular}

lengths considered here. The beam widths are $20^{\prime \prime} \times 27^{\prime \prime}$ at $28 \mu \mathrm{m}, 14^{\prime \prime} \times 27^{\prime \prime}$ at $17 \mu \mathrm{m}$, and $20^{\prime \prime} \times 33^{\prime \prime}$ at $34 \mu \mathrm{m}$. The SWS data were also reduced with the OLP version 7 and the ISAP package. No line was detected at any of the position, with typical upper limits of $\sim 1 \times 10^{-13} \mathrm{erg} \mathrm{s}^{-1} \mathrm{~cm}^{-2}$ for all lines.

\section{Mapping results}

In this section we describe the results of each observation. The nature and the properties of the various components will be discussed in Sect. 4, but to help the reader follow the discussion, the definition of the various components is briefly reviewed here (see Table 3 ). The two proto-stellar objects in the field are IRAS 16293-2422, a well documented proto-binary system (see Ceccarelli et al. 2000a; van Dishoeck et al. 1995 for recent summaries) and $16293 \mathrm{E}$, a source initially discovered through its strong ammonia emission (Mizuno et al. 1990), and whose protostellar nature is discussed by Loinard et al. (in preparation). In addition three CO peaks associated with the outflows in the region were known prior to the present paper: they were initially reported by Walker et al. (1986): the North East Red (NER) lobe at offsets $\left(90^{\prime \prime}, 50^{\prime \prime}\right)$, the West Red (WR ) lobe at $\left(-30^{\prime \prime}, 40^{\prime \prime}\right)$ and the East Red (ER) at offset $\left(20^{\prime \prime}, 30^{\prime \prime}\right)$. Recently HI2000, using a higher spatial resolution $\mathrm{SiO}$ map, renamed NER as E2, WR as W1 and W2, and ER as E1. We shall adopt the HI2000 nomenclature for the rest of the paper. In this paper, we report the 


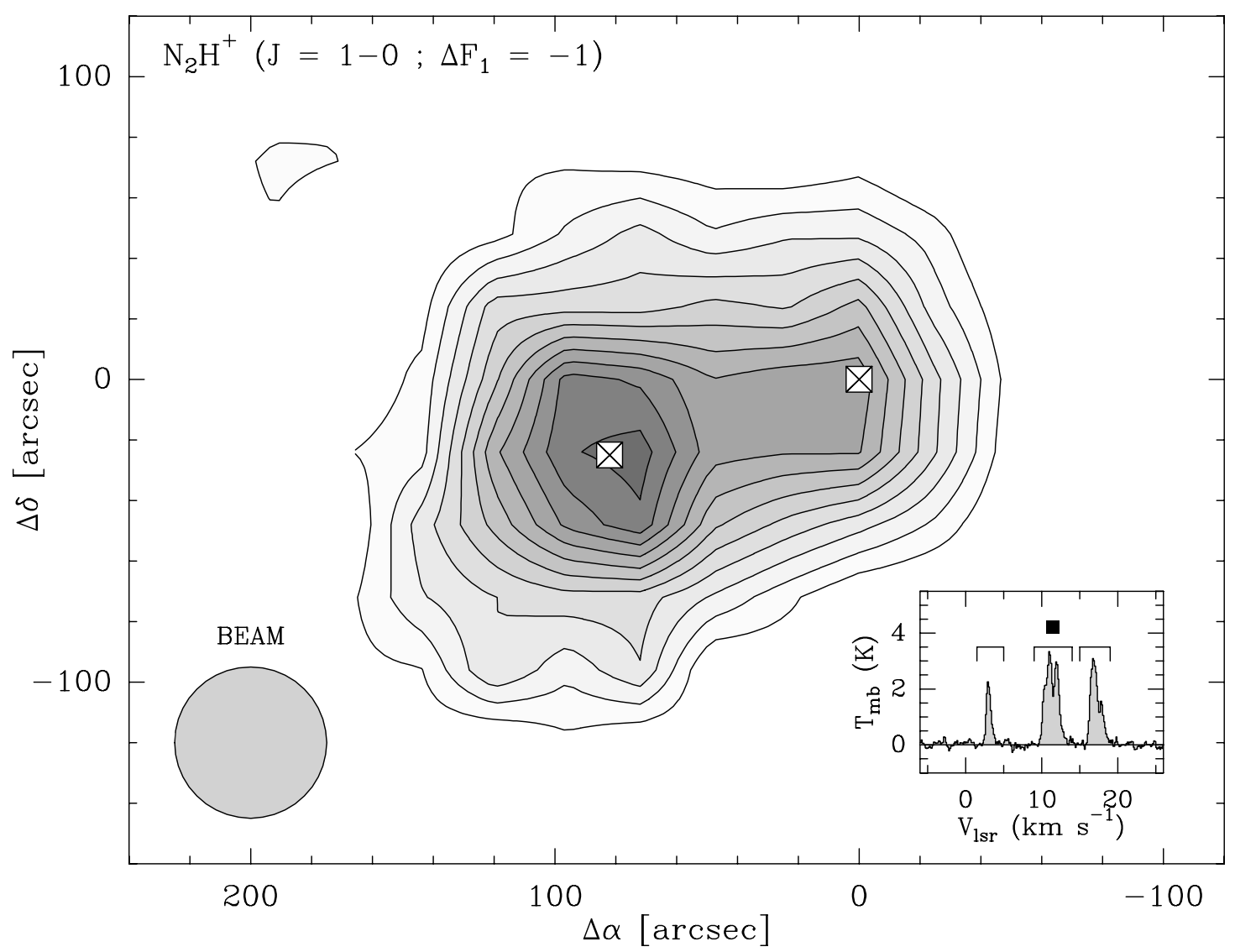

Fig. 1. $\mathrm{N}_{2} \mathrm{H}^{+} J=1 \rightarrow 0$ line integrated intensity map. The first level is at $1 \mathrm{Kkm} \mathrm{s}^{-1}$ and the level step is $0.5 \mathrm{~K} \mathrm{~km} \mathrm{~s}{ }^{-1}$. The two crossed square symbols show the position of the two protostellar sources in the field: IRAS $16293-2422$ at offsets $\left(0^{\prime \prime}, 0^{\prime \prime}\right)$, and $16293 \mathrm{E}$ at offsets $\left(+82^{\prime \prime},-25^{\prime \prime}\right)$. The integrated intensity was calculated only over the central three hyperfine components corresponding to $\Delta F_{1}=-1$, and shown by the small black square in the inset at the bottom right of the figure.

detection of two additional structures seen in $\mathrm{H}_{2} \mathrm{CO}$ (see Sect. 3.2). One of them, which we call HE1 $\left(\mathrm{H}_{2} \mathrm{CO}\right.$ East-1), could be associated with the outflow powered by the newly discovered source, 16293E (see below), and the other one (HE2 - $\mathrm{H}_{2} \mathrm{CO}$ East-2) corresponds to the terminal shock of a flow driven by one of the sources in IRAS 16293-2422.

\section{1. $\mathrm{N}_{2} \mathrm{H}^{+}$line emission}

The $J=1 \rightarrow 0$ transition of $\mathrm{N}_{2} \mathrm{H}^{+}$is split into seven hyperfine components by the interaction of the molecular electric field gradient with the electric quadrupole moments of the two nitrogen nuclei. The integrated intensity map of the central 3 components $(\Delta F=-1)$, shown in Fig. 1, clearly peaks on the two protostellar sources: IRAS $16293-2422$ at offsets $\left(0^{\prime \prime}, 0^{\prime \prime}\right)$, and $16293 \mathrm{E}$ at offsets $\left(+82^{\prime \prime},-25^{\prime \prime}\right)$. The non-linear hyperfine fitting method HFS (included in the CLASS data reduction package; Forveille et al. 1989) was applied to the $\mathrm{N}_{2} \mathrm{H}^{+}$ spectrum observed towards the $16293 \mathrm{E}$ position: it yields $3.4 \mathrm{~km} \mathrm{~s}^{-1}$ for the $16293 \mathrm{E}$ LSR velocity. The velocity of $16293 \mathrm{E}$ thus appears significantly different from that of the ambient cloud ( 3.4 versus $3.8 \mathrm{~km} \mathrm{~s}^{-1}$ ) a result that suggests that $16293 \mathrm{E}$ is physically distinct from the rest of the cloud.
Table 3. List of the various components present around IRAS 16293-2422, together with the atomic and molecular lines detected at these positions and the appropriate references. 1: Walker et al. (1986); 2: van Dishoeck et al. (1995); 3: Hirano et al. (2000); 4: this work.

\begin{tabular}{lrrlc}
\hline Position & $\begin{array}{r}\Delta \alpha \\
\left({ }^{\prime \prime}\right)\end{array}$ & $\begin{array}{r}\Delta \delta \\
\left({ }^{\prime \prime}\right)\end{array}$ & Species & Ref. \\
\hline IRAS 16293 & 0 & 0 & $\mathrm{~N}_{2} \mathrm{H}^{+}, \mathrm{H}_{2} \mathrm{CO}, \mathrm{SiO}, \mathrm{O}$ & $1,2,3,4$ \\
16293E & +82 & -25 & $\mathrm{~N}_{2} \mathrm{H}^{+}$ & 4 \\
E1 (ER) & +50 & 0 & $\mathrm{H}_{2} \mathrm{CO}, \mathrm{SiO}, \mathrm{O}$ & $2,3,4$ \\
E2 (NER) & +90 & +50 & $\mathrm{SiO}$ & 3,4 \\
W1 (WR) & -20 & +10 & $\mathrm{H}_{2} \mathrm{CO}, \mathrm{SiO}$ & $2,3,4$ \\
W2 (WR) & -20 & +60 & $\mathrm{H}_{2} \mathrm{CO}, \mathrm{SiO}$ & 3,4 \\
HE1 & +140 & -70 & $\mathrm{H}_{2} \mathrm{CO}$ & 4 \\
HE2 & +70 & -70 & $\mathrm{H}_{2} \mathrm{CO}$ & 4 \\
\hline
\end{tabular}

\section{2. $\mathrm{H}_{2} \mathrm{CO}$ line emission}

The morphology of the $\mathrm{H}_{2} \mathrm{CO}$ emission line (Fig. 2) is quite different from that of the $\mathrm{N}_{2} \mathrm{H}^{+}$emission. Four peaks are present: the strongest peak corresponds to the protostar IRAS 16293-2422 itself. It should be noted that no obvious $\mathrm{H}_{2} \mathrm{CO}$ emission enhancement is found at the position of $16293 \mathrm{E}$. The other three peaks are labeled E1, 


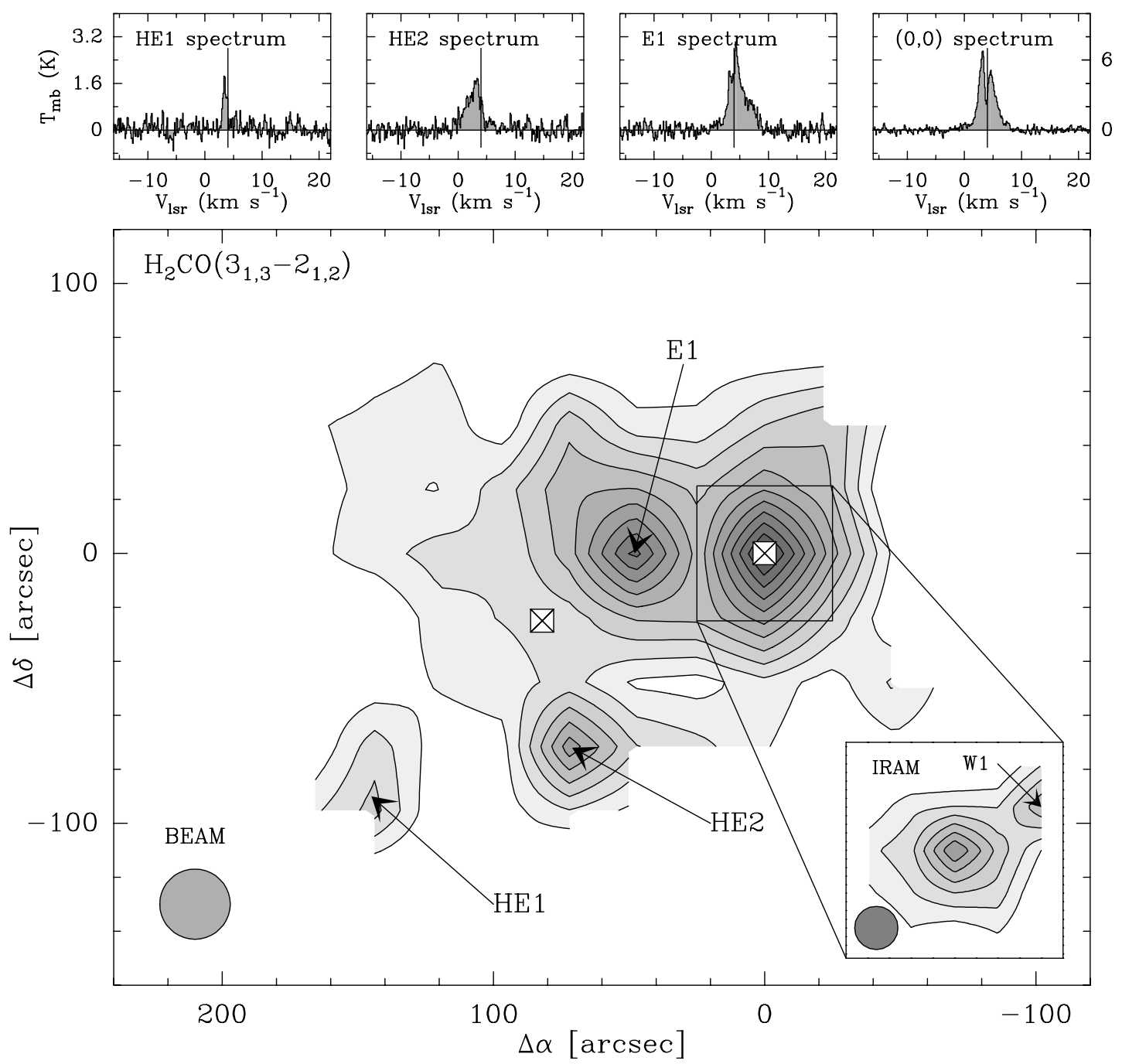

Fig. 2. Bottom: $\mathrm{H}_{2} \mathrm{CO} 3_{1,3}-2_{1,2}$ integrated intensity map. The first level is at $3.0 \mathrm{~K} \mathrm{~km} \mathrm{~s}^{-1}$, and the level step is $1.5 \mathrm{~K} \mathrm{~km} \mathrm{~s}{ }^{-1}$. The positions of the two protostars in the field are shown with the same marker as in Fig. 1, and the positions of the three main $\mathrm{H}_{2} \mathrm{CO}$ peaks are labeled (E1, HE1, and HE2). Top: spectra at the four peak positions. The intensities are in main beam brightness temperature; they range from -0.5 to $2 \mathrm{~K}$ for the three leftmost spectra, and from -1.25 to $5 \mathrm{~K}$ for the rightmost one. The vertical line on each spectrum shows the position of the systemic velocity of the cloud $\left(V_{\mathrm{lsr}}=3.8 \mathrm{~km} \mathrm{~s}{ }^{-1}\right)$.

HE1 and HE2 in Fig. 2. E1 lies close to a strong $\mathrm{SiO}$ emission peak (HI2000). The other two peaks are reported here for the first time; since they were detected through their $\mathrm{H}_{2} \mathrm{CO}$ emission, and are both located to the East of IRAS 16293-2422, we label them HE1 and HE2.

The $\mathrm{H}_{2} \mathrm{CO}$ line towards E1 is redshifted (Fig. 2) with respect to the cloud velocity, while that in HE1 is slightly blueshifted. Although E1 has been interpreted by HI2000 as part of a large flow emanating from IRAS 16293-2422, this velocity symmetry, and the almost perfect alignment between E1, HE1, and 16293E, would suggest that E1 and HE1 are rather two shocked regions resulting from the impact with the surrounding cloud, of the lobes of an outflow powered by $16293 \mathrm{E}$. However, the narrow width of the $\mathrm{H}_{2} \mathrm{CO}$ line in $\mathrm{HE} 1$ seems to be in contradiction with the interpretation of HE1 being a shock. In Fig. 3, we show $\mathrm{HCO}^{+} J=1 \rightarrow 0$ spectra obtained between HE1 and E1 through 16293E with the IRAM-30 m telescope (details of these observations are reported in Loinard et al. in preparation). Between 16293E and HE1, a blue wing is clearly seen, although at the position of HE1 itself, the wing has disappeared. North-West of $16293 \mathrm{E}$ the profiles show strong red wings which end in E1, at the position of a strong $\mathrm{SiO}$ peak (see below). This strongly suggests that 16293E drives an outflow oriented North-West - South-East with a spatial extent of about $80^{\prime \prime}$ and velocities going from $-6 \mathrm{~km} \mathrm{~s}^{-1}$ to $12 \mathrm{~km} \mathrm{~s}^{-1}$.

The fairly bright $\mathrm{H}_{2} \mathrm{CO}$ emission towards HE2 is completely blueshifted, and corresponds to a quite wide line profile (Fig. 2), suggestive of outflowing material. The high resolution map obtained with IRAM (Fig. 2) shows a red-shifted $\mathrm{H}_{2} \mathrm{CO}$ peak almost coincident with W1. Once again, the near alignment of HE2, IRAS 16293-2422 and W1, together with the red and blue line shifts would suggest that W1 and HE2 are the two lobes of an outflow 


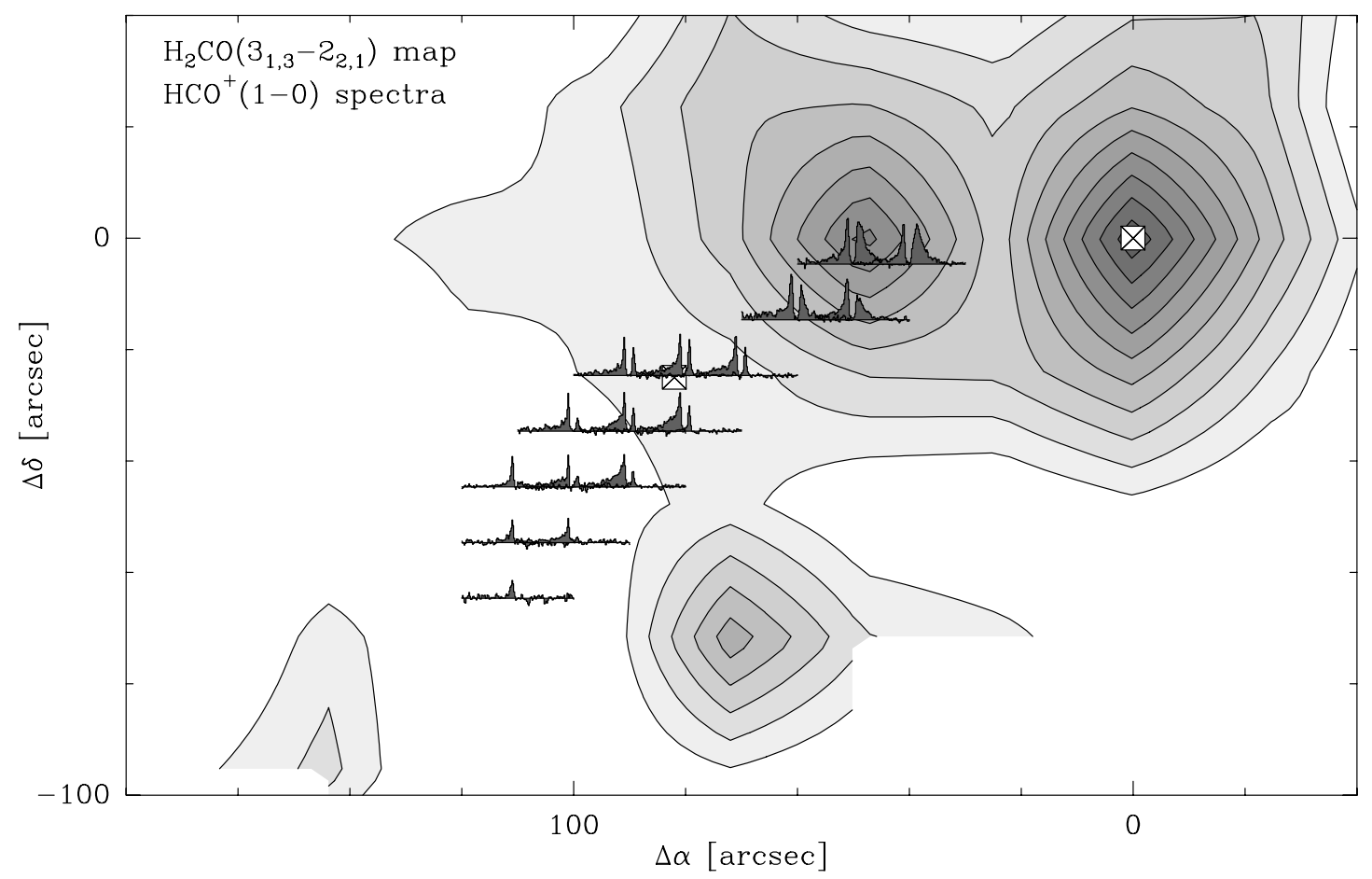

Fig. 3. $\mathrm{HCO}^{+} J=1 \rightarrow 0$ emission spectra in the region surrounding $16293 \mathrm{E}$ superimposed on part of the $\mathrm{H}_{2} \mathrm{CO}$ map (see Loinard et al. in preparation). Note that the profiles have red wings to the North-West of 16293E where E1 lies, and blue wings to the South-East towards HE1. But very close to HE1 the profiles become very narrow.
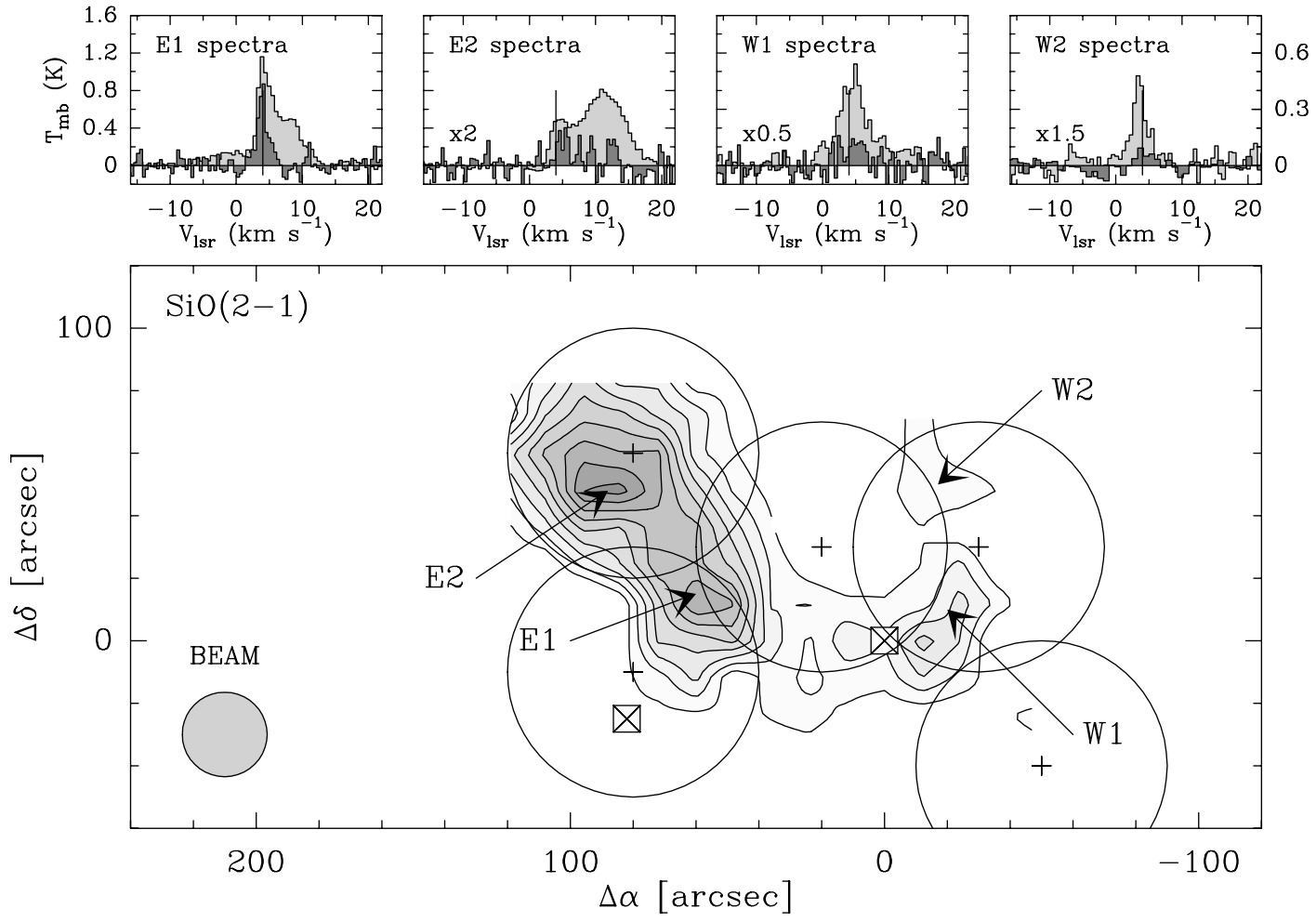

Fig. 4. Bottom: $\mathrm{SiO} J=2 \rightarrow 1$ integrated intensity map. The first level is at $1 \mathrm{~K} \mathrm{~km} \mathrm{~s}^{-1}$, and the level step is $0.75 \mathrm{Kkm} \mathrm{s}$. The positions of the two protostars in the field are shown with the same marker as in Fig. 1, and the positions of the four SiO peaks are labeled (E1, E2, W1, and W2). The positions of the ISO observations together with the ISO beam are also indicated (cf. Table 2). Top: $\mathrm{SiO} J=2 \rightarrow 1$ and $J=5 \rightarrow 4$ spectra at the four peak positions. The intensities are in main beam brightness temperature; for the $J=2 \rightarrow 1$ transition (shown in light grey) they range from -0.2 to $1.3 \mathrm{~K}$ for the E1 and E2 spectra, and from -0.1 to $0.65 \mathrm{~K}$ for the $\mathrm{W} 1$ and $\mathrm{W} 2$ ones. The $J=5 \rightarrow 4$ spectra are shown in darker gray, and have been multiplied by the amount indicated in the bottom left corner of each panel for clarity. The vertical line on each spectrum shows the position of the systemic velocity of the cloud $\left(V_{\mathrm{lsr}}=3.8 \mathrm{~km} \mathrm{~s}^{-1}\right)$. 


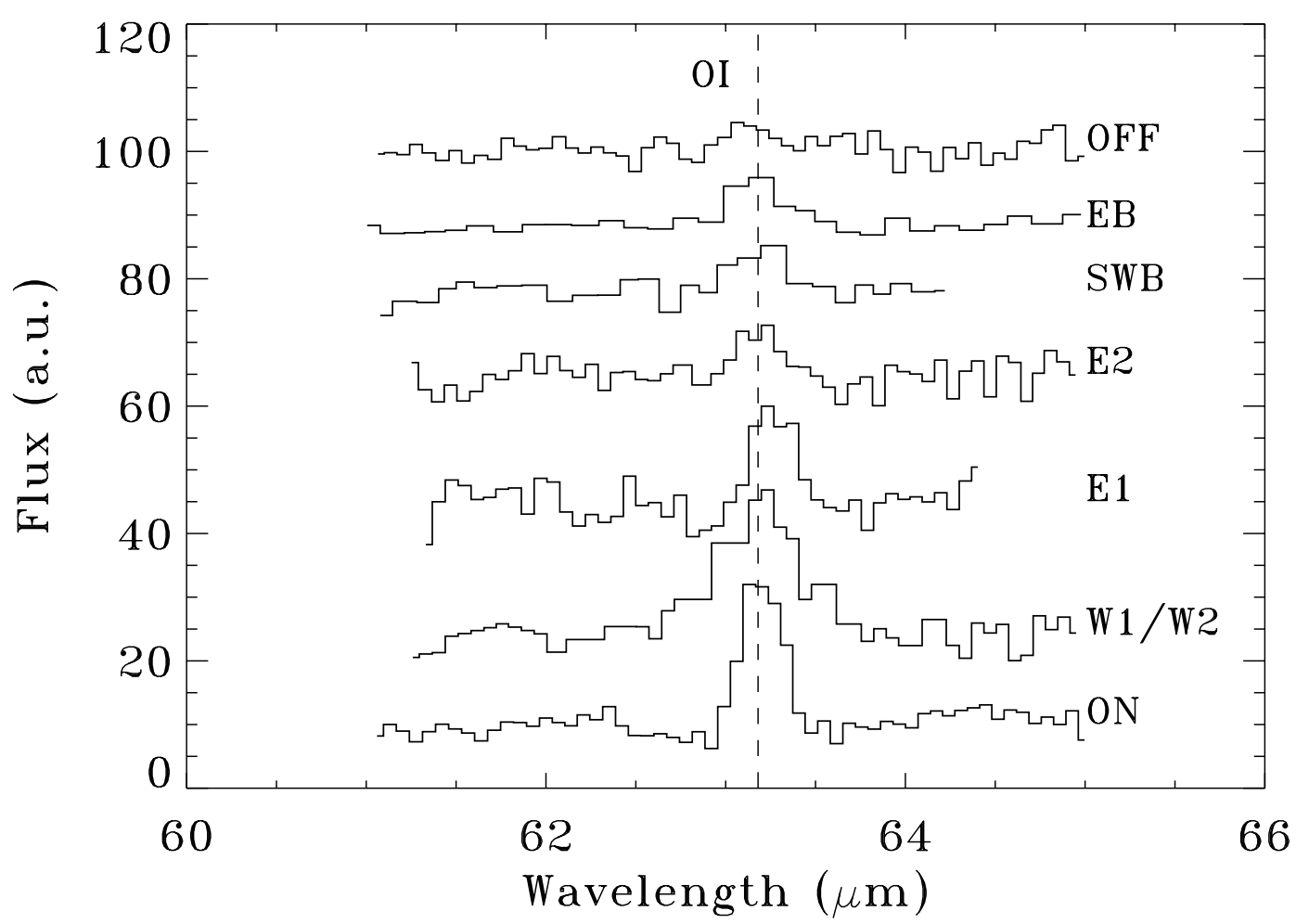

Fig. 5. [O I $63 \mu \mathrm{m}$ spectra, after continuum removal, towards the five positions along the outflows (cf. Table 2), towards IRAS 16293-2422 (ON - taken from Ceccarelli et al. 1998, but re-reduced with OLP v.7), and towards the off-outflow position (OFF - taken from Caux et al. 1999). The vertical scale is given by the spectrum of the on-source position, the other spectra have been shifted by $24,45,65,78,88$, and 100 units along the $Y$ axis. The dashed line shows the position of the [O I] $63 \mu \mathrm{m}$ line.

emanating from one of the sources forming IRAS 162932422 (cf. Sect. 4.2.3).

It should be noticed that the position of W1 seen in $\mathrm{H}_{2} \mathrm{CO}$ does seem to be slightly shifted from that seen in $\mathrm{SiO}$ by $\mathrm{HI} 2000$. We shall see below that the peaks of $\mathrm{SiO}$ and $\mathrm{H}_{2} \mathrm{CO}$ emission corresponding to $\mathrm{E} 1$ are also slightly shifted (cf. Fig. 6). Although at our spatial resolutions these shifts are only marginal, we note that Codella \& Bachiller (1999) do report significant shifts between different shock tracers.

\subsection{SiO line emission}

Figure 4 shows the integrated intensity of the $\mathrm{SiO}$ $J=2 \rightarrow 1$ emission. Since we did not detect any $\mathrm{SiO}$ emission in the southern part of the region, the figure displays only the northern part of the $\mathrm{SiO}$ emission. Although they have features in common, this $\mathrm{SiO}$ map differ from the $\mathrm{N}_{2} \mathrm{H}^{+}$and $\mathrm{H}_{2} \mathrm{CO}$ ones. An equivalent $\mathrm{SiO} J=2 \rightarrow 1$ map with similar spatial resolution but with a slightly smaller extent has been recently published by HI2000. In their paper, HI2000 give a full description of the spatial distribution of the $\mathrm{SiO} J=2 \rightarrow 1$ emission. For comparison with our $\mathrm{SiO} J=3 \rightarrow 2$ and $J=5 \rightarrow 4$ observations, we summarize here the main features of the $J=2 \rightarrow 1$ emission. It presents four distinct peaks, E1, E2, W1 and W2. The lines (Fig. 4) at E1, E2, and - to a smaller extent - at W1 are red-shifted with respect to the systemic cloud ve- locity $\left(3.8 \mathrm{~km} \mathrm{~s}^{-1}\right)$, while the line at $\mathrm{W} 2$ is blue-shifted, but quite narrow. Weak $\mathrm{SiO} J=2 \rightarrow 1$ emission is detected towards IRAS 16293-2422, as already reported by Ceccarelli et al. (2000a).

In the $J=3 \rightarrow 2$ line emission map (not presented here) we find the same conspicuous peaks as in Fig. 4: E1, E2, W1 and W2. IRAS 16293-2422 is also weakly seen in the $\mathrm{SiO} J=3 \rightarrow 2$ emission. Conversely, we detected $\mathrm{SiO} J=5 \rightarrow 4$ emission only in a few points of the map, notably around IRAS 16293-2422, E1 and marginally towards E2 and W1. Figure 4 shows the spectra at these positions of $\mathrm{SiO} J=2 \rightarrow 1$ and $J=5 \rightarrow 4$. Note that the $J=5 \rightarrow 4$ emission is very weak in the wings of the lines and more pronounced around the systemic velocities, especially towards E1. The line at E1 is slightly red-shifted compared to the systemic velocity of IRAS 16293-2422, but it is quite red-shifted with respect to the systemic velocity of $16293 \mathrm{E}\left(3.4 \mathrm{~km} \mathrm{~s}^{-1}\right.$ - Sect. 3.1). Following the discussion of Sect. 3.2, we argue that the $\mathrm{SiO}$ emission at E1 is due to a shock associated with the impact of the red lobe of the outflow powered by $16293 \mathrm{E}$.

\subsection{Atomic oxygen line emission}

Atomic oxygen was detected at all the positions observed towards the outflows (Fig. 5), with emission well in excess of the diffuse emission measured in the OFF position (Table 2). Besides the protostellar source 


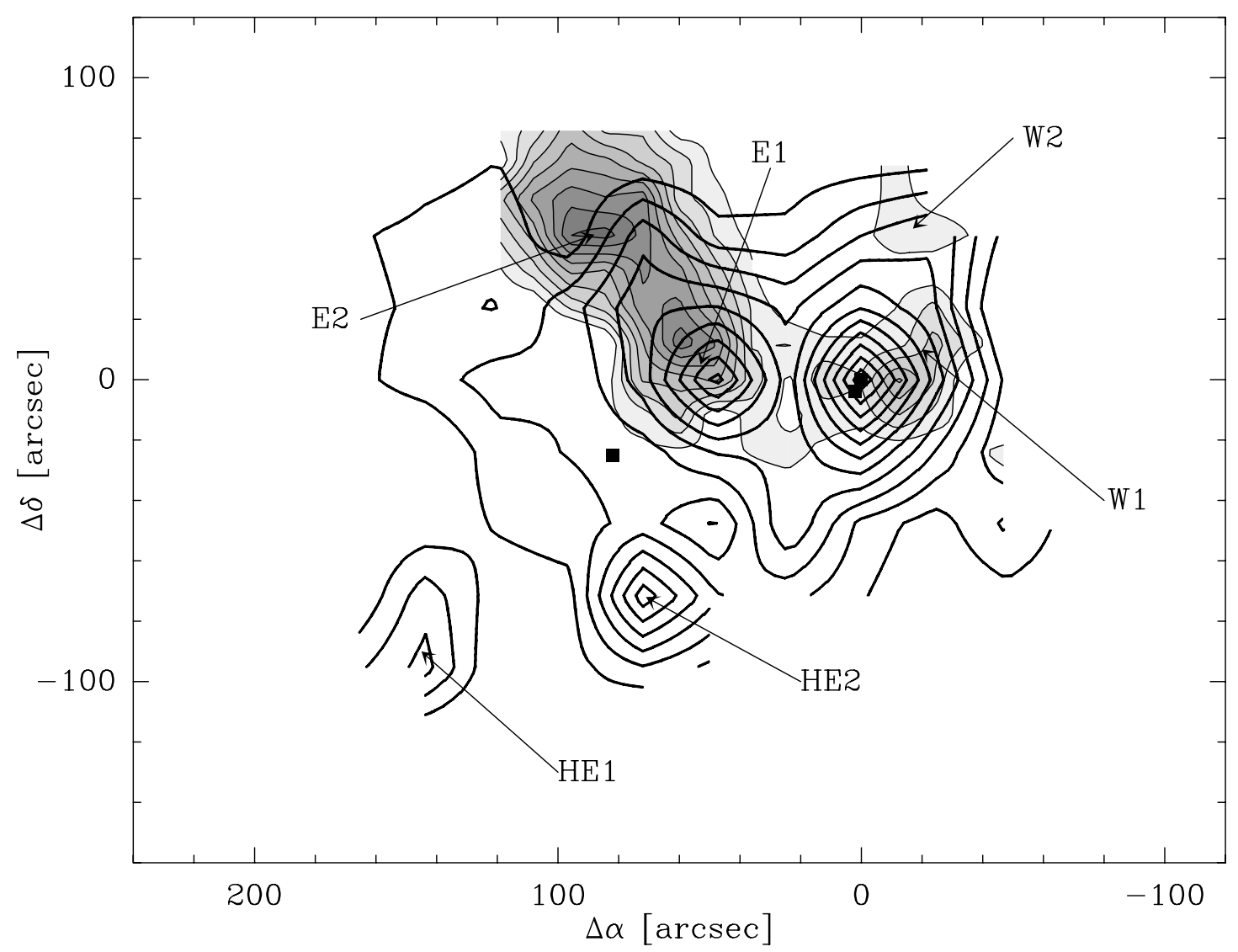

Fig. 6. Superposition of the $\mathrm{SiO}: 2 \rightarrow 1$ and $\mathrm{H}_{2} \mathrm{CO}: 3_{13}-2_{12}$ integrated intensity maps. Note that the beamsizes $\left(26^{\prime \prime}\right)$ are similar for the two transitions. The levels are those indicated in the corresponding figures (Figs. 2 and 4). The position of the three protostars in the field, 16293A, 16293B and 16293E, are indicated by black squares. The positions of the various regions discussed in the text are labeled accordingly.

IRAS 16293-2422, the strongest [O I] emission is detected towards the $\mathrm{W} 1 / \mathrm{W} 2$ peak. [O I] emission $\sim 40^{\prime \prime}$ West of IRAS 16293-2422 (at the position of W2) had already been reported by Ceccarelli et al. (1997) using data from the Kuiper Airborne Observatory (KAO). The flux reported by Ceccarelli et al. $(1997)-(9.5 \pm 2.4) \times$ $10^{-12} \mathrm{erg} \mathrm{s}^{-1} \mathrm{~cm}^{-2}$ - is consistent with that found here, implying that the emission originates in a region smaller than the $40^{\prime \prime} \mathrm{KAO}$ beam. Ionized carbon was also detected in all positions at a level comparable with that measured around the flow, i.e. $4 \times 10^{-12} \mathrm{erg} \mathrm{s}^{-1} \mathrm{~cm}^{-2}$ (Caux et al. 1999), suggesting that it originates in the cloud skin illuminated by the interstellar UV field (Ceccarelli et al. 1998). Finally several other important molecular transitions lie in the wavelength range observed by ISO, notably $\mathrm{H}_{2} \mathrm{O}, \mathrm{CO}$ and $\mathrm{OH}$ (e.g. Ceccarelli et al. 1998). Along the outflow we did not detect any line from these molecules, setting an upper limit on their fluxes of $2 \times 10^{-12} \mathrm{erg} \mathrm{s}^{-1} \mathrm{~cm}^{-2}$.

\section{Discussion}

The data presented in Sect. 3 show emission line peaks coinciding with the protostars IRAS $16293-2422$ and $16293 \mathrm{E}$, with four positions associated with the outflows E1, E2, HE2 and W1, and with two additional positions,
HE1 and W2. In this section, we discuss first the two protostars, and then the various other peaks.

\subsection{Protostellar sources}

\subsubsection{IRAS 16293-2422}

IRAS $16293-2422$ is very strong in [O I] $63 \mu \mathrm{m}$ and $\mathrm{H}_{2} \mathrm{CO}$, moderately strong in $\mathrm{N}_{2} \mathrm{H}^{+}$, and hardly visible in $\mathrm{SiO}$. The $\mathrm{H}_{2} \mathrm{CO} J=3_{1,3} \rightarrow 2_{1,2}$ line on-source (Fig. 2) shows a deep absorption feature at the systemic velocity of the source, suggestive of self-absorption. The $\mathrm{H}_{2}^{13} \mathrm{CO}$ observations presented by Loinard et al. (2000), however, show that the $\mathrm{H}_{2} \mathrm{CO}$ is only moderately optically thick. The absorption feature is, thus, most likely due to cold foreground material. Using $\mathrm{H}_{2} \mathrm{CO}$ multi-frequency observations, Ceccarelli et al. (2000b) showed that the $\mathrm{H}_{2} \mathrm{CO}$ emission in IRAS 16293-2422 originates from two regions: a warm $(T \geq 100 \mathrm{~K})$ small central core $(r \leq 150 \mathrm{AU})$ where the $\mathrm{H}_{2} \mathrm{CO}$ abundance is high $\left(1.0 \times 10^{-7}\right)$, and an extended, relatively cool surrounding envelope where the abundance is fairly low $\left(1.1 \times 10^{-9}\right)$. In both regions, $\mathrm{H}_{2} \mathrm{CO}$ is desorbed by grain mantles. The different abundances reflect the $\mathrm{H}_{2} \mathrm{CO}$ formation on polar (hot core) and non-polar (outer envelope) ices (Ceccarelli et al. 2001). 
The $\mathrm{SiO}$, [O I] and $\mathrm{H}_{2} \mathrm{O}$ lines emitted by IRAS $16293-$ 2422 were also analyzed by Ceccarelli et al. (2000a). They all originate in the two regions identified by Ceccarelli et al. (2000b). In particular, while the $\mathrm{SiO}$ $J=2 \rightarrow 1$ emission is dominated by the cold outer envelope emission, where the $\mathrm{SiO}$ abundance is low $\left(\sim 10^{-12}\right)$, higher $\mathrm{SiO}$ transitions (such as the $J=5 \rightarrow 4$ ) probe the inner warmer regions, where the $\mathrm{SiO}$ abundance is high $\left(\sim 10^{-8}\right)$. All these observations show that IRAS 162932422 possesses a "hot core" - like region, extending to about $150 \mathrm{AU}$ from the protostar center. In that region, the dust mantles evaporate, injecting large amounts of $\mathrm{H}_{2} \mathrm{O}, \mathrm{SiO}$ and $\mathrm{H}_{2} \mathrm{CO}$, previously stored onto the mantles, back to the gas phase.

\subsubsection{E}

$16293 \mathrm{E}$ shows a trend almost exactly opposite to that of IRAS 16293-2422: it is weak in $\mathrm{H}_{2} \mathrm{CO}$ and [O I] $63 \mu \mathrm{m}$, but strong in $\mathrm{N}_{2} \mathrm{H}^{+}$. No $\mathrm{SiO}$ emission is seen at this position. Since the $\mathrm{N}_{2} \mathrm{H}^{+} J=1 \rightarrow 0$ transition is optically thin in all hyperfine components, its intensity is essentially proportional to its column density. IRAS 16293-2422 is about 6 times brighter in the millimeter continuum than $16293 \mathrm{E}$, so the difference in $\mathrm{N}_{2} \mathrm{H}^{+}$emission clearly points to different abundances.

Chemical models of dense molecular clouds predict that $\mathrm{N}_{2} \mathrm{H}^{+}$is mostly formed by ion-molecule reactions, which proceed quickly at low temperatures. The major production route is through the reaction: $\mathrm{H}_{3}^{+}+\mathrm{N}_{2} \rightarrow$ $\mathrm{N}_{2} \mathrm{H}^{+}+\mathrm{H}_{2}$ (Womack et al. 1992), whereas the major destruction route involves $\mathrm{CO}$. Consequently, $\mathrm{N}_{2} \mathrm{H}^{+}$is believed to be efficiently destroyed in dense $\left(n\left(\mathrm{H}_{2}\right)>\right.$ $\left.10^{6} \mathrm{~cm}^{-3}\right)$ and warm $\left(T_{\mathrm{k}}>100 \mathrm{~K}\right)$ environments, but to be a good tracer of extended, cold, and quiescent material (Turner \& Thaddeus 1977; Womack et al. 1992; Turner 1995; Caselli et al. 1995). The higher $\mathrm{N}_{2} \mathrm{H}^{+}$abundance towards $16293 \mathrm{E}$ thus likely reflects its lower temperature.

Using far-IR, and sub-millimetric continuum observations, Loinard et al. (in prep.) do indeed show that the dust temperature in $16293 \mathrm{E}\left(T_{\text {dust }}=24 \mathrm{~K}\right)$ is significantly lower than in IRAS $16293-2422\left(T_{\text {dust }}=45 \mathrm{~K}\right)$. Those data also show that the fraction of continuum emission emitted longwards of $350 \mu \mathrm{m}$ in 16293E is well in excess to the canonical limit for Class 0 sources (André et al. 1993). In addition we have indicated in paragraph 3.2 that $16293 \mathrm{E}$ drives a well-defined outflow whose northern lobe impacts the ambient cloud in E1. 16293E can therefore be argued to be a bona fide Class 0 protostar, whose bolometric luminosity is calculated to be $\sim 2.3 L_{\odot}$ (Loinard et al., in prep.).

It is intriguing that a cold gas condensation like $16293 \mathrm{E}$ is not visible in $\mathrm{H}_{2} \mathrm{CO}$. That the doubly deuterated form of formaldehyde $\left(\mathrm{D}_{2} \mathrm{CO}\right)$ and ammonia $\left(\mathrm{ND}_{2} \mathrm{H}\right)$, has been detected towards that source (Loinard et al. 2001) makes the absence of conspicuous $\mathrm{H}_{2} \mathrm{CO}$ emission even more surprising. It should be noted, however, that the whole region is bright in $\mathrm{H}_{2} \mathrm{CO}$ : at the position of IRAS 16293-2422, the peak main beam temperature of the $J=3_{1,3}-2_{1,2}$ transition is about $6 \mathrm{~K}\left(\int T_{\mathrm{mb}} \mathrm{d} v \simeq 17.5 \mathrm{~K} \mathrm{~km} \mathrm{~s}^{-1}\right)$, and although there is no clear excess emission at the position of $16293 \mathrm{E}$, the brightness there is still over $2 \mathrm{~K}$ $\left(\int T_{\mathrm{mb}} \mathrm{d} v \simeq 5.0 \mathrm{~K} \mathrm{~km} \mathrm{~s}^{-1}\right)$. Even if $16293 \mathrm{E}$ did emit significantly in $\mathrm{H}_{2} \mathrm{CO}$, its location in a region that is overall $\mathrm{H}_{2} \mathrm{CO}$ rich would make it difficult for $16293 \mathrm{E}$ to stand out. Recall that IRAS $16293-2422$ is about 6 times brighter in the millimetric continuum than 16293E: for similar $\mathrm{H}_{2} \mathrm{CO}$ abundances, IRAS 16293-2422 would easily stand out, while $16293 \mathrm{E}$ would not. The lower gas density and temperature of $16293 \mathrm{E}$ can also contribute to the relatively lower $\mathrm{H}_{2} \mathrm{CO}$ line emission. Finally the relatively large beam of the SEST observations can also contribute to smooth the $16293 \mathrm{E}$ emission.

\subsection{Outflows}

It is interesting that $\mathrm{SiO}, \mathrm{H}_{2} \mathrm{CO}$ and [OI] $63 \mu \mathrm{m}$ emission, all of them usually considered as good tracers of shocks, have such different morphologies in the region around IRAS 16293-2422. To facilitate their comparison we present in Fig. 6 a superposition of the $\mathrm{SiO}$ and $\mathrm{H}_{2} \mathrm{CO}$ maps. There are two positions where $\mathrm{SiO}$ and $\mathrm{H}_{2} \mathrm{CO}$ molecules seem to co-exist in large amounts, E1 and, to a smaller extent, W2. In W2 the [O I] $63 \mu \mathrm{m}$ emission is also strong, and this is the only region of the area where all three shocks tracers peak. Other regions, like HE1, HE2 and $\mathrm{E} 2$, are either prominent in $\mathrm{H}_{2} \mathrm{CO}$ or $\mathrm{SiO}$ emission, but not in both. It is therefore clear that each molecular species seems to trace different regions. In this section, we will discuss individually each of these regions in our map, in an attempt to understand just how different they are.

\subsubsection{Emission peak E2}

This is the strongest $\mathrm{SiO}$ peak. It corresponds to the far end of the North-East lobe of the outflow powered by IRAS 16293-2422, in coincidence with the CO peak called NER by Walker et al. (1986). Note that no strong SiO emission is detected towards the corresponding $\mathrm{CO}$ blue lobe to the South-West (called SWB by Walker et al.), suggesting that IRAS 16293-2422 is not very deeply embedded in the cloud and that the blue lobe exits from the cloud before having a strong interaction with it. The system HE2-W1 seems to show a similar behavior as we shall see in Sect. 4.2.3.

At position E2 the bulk of the $J=2 \rightarrow 1$ emission (and the $J=3 \rightarrow 2$ as well) is very red-shifted (Fig. 4 ), but a component at ambient velocity is also seen, mostly in the $J=5 \rightarrow 4$ transition. While the high velocity component $\left(v \geq 8 \mathrm{~km} \mathrm{~s}^{-1}\right)$ is only present at the end of the outflow, the "ambient" component is detected all across the North lobe of the outflow, and appears to be strongest near its South border as already noted by HI2000 (see the $\mathrm{SiO}$ channel map [Fig. 2] in HI2000). The spectra 


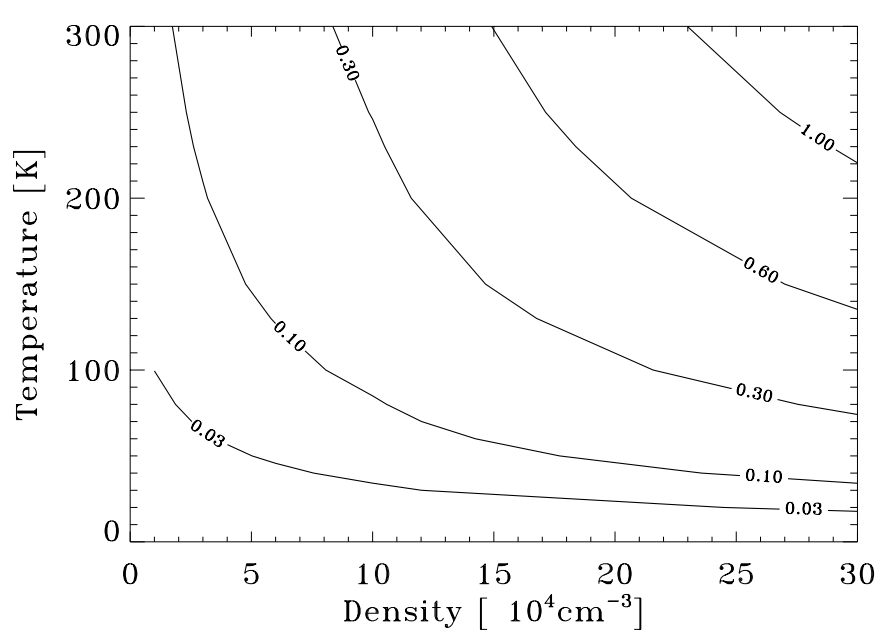

Fig. 7. $\mathrm{SiO} 5 \rightarrow 4$ over $2 \rightarrow 1 T_{\mathrm{mb}}$ computed ratios as function of temperature and density. In these computations the lines are assumed optically thin. The observed $5-4$ over $2-1 T_{\mathrm{mb}}$ ratios are 0.1 for the high velocity component in E1 and E2 and 0.6 and 0.3 for the low velocity component in E1 and E2 respectively.

shown in Fig. 4 clearly demonstrate that the $J=5 \rightarrow 4$ to $J=2 \rightarrow 1$ line ratios are quite different for the high and the ambient velocity components. Quantitatively this ratio appears to be about 0.1 for the high velocity gas, but about 3 times higher for the ambient component (recall that since the original $\mathrm{SiO} J=5 \rightarrow 4$ data are not Nyquist-sampled, the intensities obtained here could be affected by slight errors [see Sect. 2.1]). This implies that the low velocity component and high velocity component have different temperature and/or density. Hence not only the two components differ kinematically but also physically. Therefore the interpretation offered by HI2000 in terms of differing projection effects appears quite unlikely. On the contrary, the two $\mathrm{SiO}$ emission peaks probe two physically distinct components of the outflow.

To better characterize the physical parameters of these two components, we modeled the $\mathrm{SiO}$ line intensities using an LVG code. Details of this code are given in Ceccarelli et al. (2000a). We compute theoretical ratios of the $\mathrm{SiO} J=5 \rightarrow 4$ over $J=2 \rightarrow 1 \int T_{\mathrm{mb}} \mathrm{d} v$ as a function of temperature and density, assuming optically thin lines. The results of this computation show that the $J=2 \rightarrow 1$ and $J=5 \rightarrow 4$ lines cannot constraint efficiently the temperature and density of the emitting gas (Fig. 7). However the ambient velocity component has to be warmer than $\sim 70 \mathrm{~K}$ and denser than $\sim 8 \times 10^{4} \mathrm{~cm}^{-3}$, while the high velocity component has to be either somewhat colder or, more likely, significantly less dense than the ambient component. The corresponding $\mathrm{SiO}$ column densities are $\sim 6 \times 10^{13} \mathrm{~cm}^{-2}$ (adopting $n\left(\mathrm{H}_{2}\right)=10^{4} \mathrm{~cm}^{-3}$ and $\left.T_{k}=100 \mathrm{~K}\right)$ and $\sim 4 \times 10^{12} \mathrm{~cm}^{-2}\left(n\left(\mathrm{H}_{2}\right)=10^{5} \mathrm{~cm}^{-3}\right.$ and $\left.T_{k}=150 \mathrm{~K}\right)$ for the high velocity and the ambient velocity components respectively.

It should be noticed, however, that the different $\mathrm{SiO}$ line ratios could also be due to different degrees of clumpi- ness. For instance, if the $\mathrm{SiO} 5 \rightarrow 4$ emission in the high velocity component was three times more clumpy than that in the low velocity component, the physical conditions would be similar in both. Also, if the $5 \rightarrow 4$ emission originates in small clumps $2^{\prime \prime} \times 2^{\prime \prime}$ in size or even smaller, (i.e. with a filling factor smaller than 0.1) the $\mathrm{SiO}$ emission could become optically thick. In such a case, column densities and abundances could, obviously, not be estimated. However, both cases seems rather unlikely, given that the $\mathrm{SiO}$ emission is relatively widespread. Therefore the fact that the low and high velocity components are physically different is a robust result.

What is the origin of these two components? We suggest that the widespread, ambient component, which permeates the entire outflow lobe, corresponds to the shocked material belonging to the cloud, i.e. probes what is usually referred to as the cloud shock (e.g. Hollenbach 1998). In contrast the high-velocity component probes the so-called "wind shock", corresponding to the impact of the jet/wind emitted by the protostar with the ambient gas. Although it has a ten times lower volume density, the $\mathrm{SiO}$ column density of the wind shocked material, traced by the high velocity component, is more than ten times larger than that of the ambient component. This agrees well with the view that $\mathrm{SiO}$ is mainly formed by silicium released from the grain mantles and/or grains cores because of energetic shocks (e.g. Caselli et al. 1997; Schilke et al. 1997). The larger the shock velocity, the larger the amount of silicium released in the gas phase, and the larger the $\mathrm{SiO}$ abundance.

A possible reason why $\mathrm{E} 2$ does not appear in $\mathrm{H}_{2} \mathrm{CO}$ and [O I] $63 \mu \mathrm{m}$, although it is so bright in $\mathrm{SiO}$, will be discussed in Sect. 4.3.

\subsubsection{Emission peaks $\mathrm{E} 1$ and $\mathrm{HE} 1$}

The second most prominent $\mathrm{SiO}$ peak is E1, which coincides with a strong $\mathrm{H}_{2} \mathrm{CO}$ emission peak. Although it is clearly not on the line joining IRAS 16293-2422 and E2, E1 has usually been believed to be part of the North-East lobe that terminates at E2 (e.g. HI2000). Both the $\mathrm{H}_{2} \mathrm{CO}$ and the $\mathrm{SiO}$ emission are red-shifted with respect to the ambient velocity (Figs. 2 and 4). As previously mentioned, E1 may be the result of the impact into the surrounding cloud of the red lobe of an outflow powered by $16293 \mathrm{E}$. In this picture the corresponding blue lobe is elongated in the direction of the $\mathrm{HE} 1$ condensation, seen in $\mathrm{H}_{2} \mathrm{CO}$ (Fig. 3), and HE1 could be the result of the impact of this blue lobe with the cloud. In favor of this interpretation there is the alignment of the two blobs (E1 and HE1) with 16293E and the presence of an outflow emanating from 16293E as testified by the $\mathrm{HCO}^{+}$observations (Sect. 3.2). However this interpretation has a difficulty in explaining the relatively narrow line profile $\left(\sim 2 \mathrm{~km} \mathrm{~s}^{-1}\right)$ at the HE1 position.

The $\mathrm{SiO} J=5 \rightarrow 4$ to $J=2 \rightarrow 1$ line ratio in $\mathrm{E} 1$ $(\sim 0.6)$ is the highest in the map, and implies for the ambient component a density larger than $1.0 \times 10^{5} \mathrm{~cm}^{-3}$. 
This means that the clump of gas emitting at the E1 position is denser and/or warmer than the gas of the low velocity component of the E2 peak. Furthermore, the $\mathrm{SiO}$ column density for the ambient component is about $8 \times 10^{12} \mathrm{~cm}^{-2}$ (adopting $n\left(\mathrm{H}_{2}\right)=10^{5} \mathrm{~cm}^{-3}$ and $T_{k}=$ $150 \mathrm{~K}$ ), similar to that observed in $\mathrm{E} 2$. The $\mathrm{SiO}$ column density for the high velocity component is slightly smaller than the one found in E2, i.e. $\sim 5 \times 10^{13} \mathrm{~cm}^{-2}$. Then the total $\mathrm{SiO}$ column density in the direction of $\mathrm{E} 1$ is equal to $5.8 \times 10^{13} \mathrm{~cm}^{-2}$ equivalent to the one given in HI2000.

At the E1 position we also observed $\mathrm{C}^{18} \mathrm{O} J=1 \rightarrow 0$ with the SEST telescope: the line is narrow $(F W H M=$ $\left.1 \mathrm{~km} \mathrm{~s}^{-1}\right)$ and intense $\left(T_{\mathrm{mb}}=6.0 \mathrm{~K}\right)$ in the $43^{\prime \prime}$ beam of the observation, implying that it is probably dominated by the cloud gas. Assuming the line is optically thin, the ${ }^{16} \mathrm{O}$ over ${ }^{18} \mathrm{O}$ ratio equals to 490 and assuming the values for the density and the temperature derived from the $\mathrm{SiO}$ observations, we derive a $\mathrm{CO}$ column density equal to $\sim 5 \times 10^{18} \mathrm{~cm}^{-2}$. Since this is an upper limit to the $\mathrm{CO}$ column density of the gas emitting $\mathrm{SiO}$, we obtain a lower limit to the ratio $[\mathrm{SiO}] /[\mathrm{CO}]$ equal to $\sim 2 \times 10^{-6}$, and given the CO abundance in L1689N, $\left(10^{-5}\right.$ - Caux et al. 1999), a corresponding $[\mathrm{SiO}] /\left[\mathrm{H}_{2}\right] \geq 2 \times 10^{-11}$ for the ambient component. Although this is a low value, this is significantly higher than the upper limit to the $\mathrm{SiO}$ abundance in cold clouds $\left(\leq 10^{-12}\right.$; Ziurys et al. 1989; MartinPintado et al. 1992), supporting the hypothesis that E1 corresponds to shocked gas. As a final remark, note that the $\mathrm{H}_{2} \mathrm{CO}$ and $\mathrm{SiO}$ emissions are slightly shifted by $14^{\prime \prime}$ (Fig. 6). Several authors have already noticed such a shift between different shock tracers (see for example Codella \& Bachiller 1999) arguing of a different chemical behavior all along the outflow and reflecting its evolutionary stage. Since in our case, both maps have a $27^{\prime \prime}$ spatial resolution this shift is, however, only marginally significant.

The blue lobe of the outflow powered by $16293 \mathrm{E}$ is not seen in SiO. A possible explanation is that the shocked region is small $\left(\leq 10^{\prime \prime}\right)$ and the emission consequently too diluted to be detected in our SEST observations. Another possibility is that, as in the case of the blue lobe of the large scale outflow E2-SWB (see previous paragraph), the blue lobe emerges from the cloud impacting much less material. This hypothesis is discussed in more detail in the next paragraph.

\subsubsection{Emission peaks HE2 and W1}

Like HE1, HE2 is bright in $\mathrm{H}_{2} \mathrm{CO}$, but not detected in $\mathrm{SiO}$. The $\mathrm{H}_{2} \mathrm{CO}$ line in HE2 is completely blue-shifted and quite wide (Fig. 2), clearly indicative of outflowing material. As argued in Sect. 3.2 HE2 is probably the blue lobe of an outflow, presumably powered by the A components of IRAS 16293-2422, whose red counterpart may be W1, also detected in $\mathrm{SiO}$. The identification of $\mathrm{W} 1$ as the red counterpart of HE2 is supported by the rough alignment of the two blobs with the A component of the binary system IRAS 16293-2422. Unfortunately the envelope surround- ing the IRAS 16293-2422 system emits also in the $\mathrm{SiO}$ and $\mathrm{H}_{2} \mathrm{CO}$ transitions (see Ceccarelli et al. 2000a,b) and it is therefore difficult to disentangle it from the outflowing gas emission. Specifically, this blending of the two components may be responsible for the non perfect alignment. It can be noticed that there is also a slight misalignment (only marginally significant) between the $\mathrm{SiO}$ and $\mathrm{H}_{2} \mathrm{CO}$ emission peaks.

There are two difficulties with the interpretation of HE2-W1 and HE1-E1 as outflows driven by IRAS 162932422 and 16293E, respectively. First, W1 and E1 are closer to IRAS 16293-2422 and 16293E respectively than HE2 and HE1. And second, W1 and E1 are strong SiO emitters, whereas HE2 and HE1 are not. If the ejection events that produced HE2 and W1, on the one hand, and HE1 and $\mathrm{E} 1$ on the other, were simultaneous, then the lack of $\mathrm{SiO}$ emission in HE1 and HE2 has to reflect differing physical and/or chemical conditions of the shocked material. As already suggested above, a possibility is that both IRAS $16293-2422$ and $16293 \mathrm{E}$ are located close to the edge of L1689N facing us and power bipolar outflows that, on one side move toward the interior of $\mathrm{L} 1689 \mathrm{~N}$, while on the other side quickly escape from that cloud. In this scenario, E1 and W1 would correspond to the impact of the outflows with the dense gas of L1689N, whereas HE1 and HE2 impact on much less dense gas. The difference in $\mathrm{SiO}$ between the red-shifted (inner cloud material) and blueshifted (outer cloud material) lobes might then reflect differing compositions of the grain mantles in a dense and a more tenuous medium. Alternatively it might be due to the fact that the impact of an outflow with dense gas results in the partial destruction of the grain themselves (where Si would be trapped), whereas the impact on less dense gas would leave the grain cores intact. In both cases the mantles (where $\mathrm{H}_{2} \mathrm{CO}$ is trapped) would be sputtered (Schilke et al. 1997).

\subsubsection{Emission peak W2}

W2 is best seen in $\mathrm{SiO}$, but can also be identified as a shoulder to the North-West of IRAS 16293-2422 in the $\mathrm{H}_{2} \mathrm{CO}$ map. The $\mathrm{H}_{2} \mathrm{CO}$ and $\mathrm{SiO}$ lines there are mainly blue-shifted (centered at $\sim 3.7 \mathrm{~km} \mathrm{~s}^{-1}$ ) and are narrow ( $\leq 3 \mathrm{~km} \mathrm{~s}^{-1}$; Fig. 4). The $\mathrm{SiO} J=5 \rightarrow 4 / J=2 \rightarrow 1$ ratio is $\sim 0.3$ suggesting a relatively dense $\left(\geq 10^{5} \mathrm{~cm}^{-3}\right)$ and warm gas ( $\geq 80 \mathrm{~K}$; see Fig. 7 ). The $\mathrm{SiO}$ column density is $\sim 1.4 \times 10^{11} \mathrm{~cm}^{-2}$ (adopting $n\left(\mathrm{H}_{2}\right)=3 \times 10^{5} \mathrm{~cm}^{-3}$ and $T_{k}=80 \mathrm{~K}$ ). The origin of the W2 peak is not clear, and the association with an outflow in the region is not obvious, lacking a red-shifted counterpart. Nevertheless, it is clear that W2 is a place where plenty of gas phase molecules emit. It is possible that the red-shifted counterpart is heavily embedded in the cloud and therefore masked by the strong emission from the massive envelope surrounding IRAS 16293-2422. Another possibility, however, is that W2 is another very embedded source and that the molecular emission is associated with its envelope 


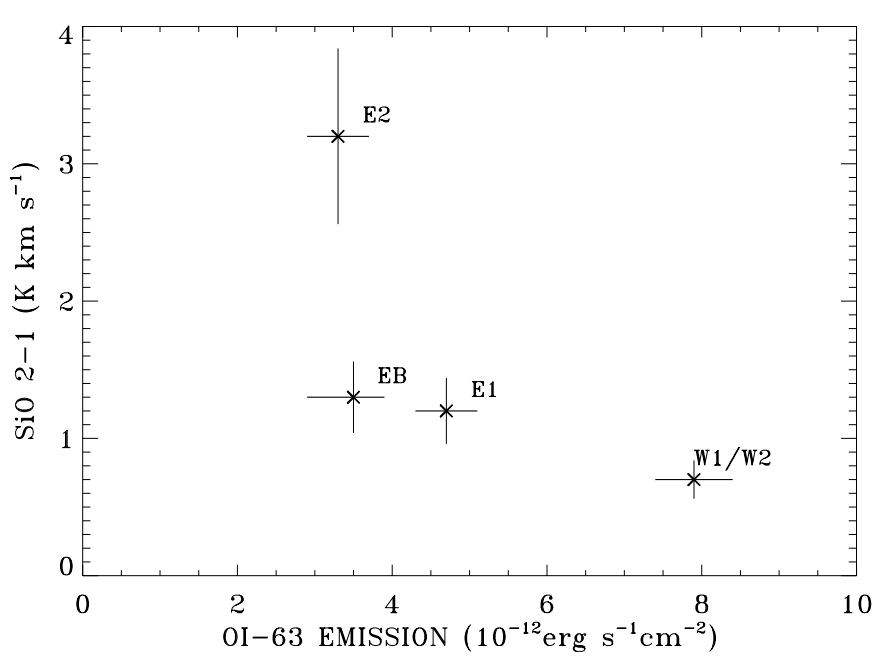

Fig. 8. Intensity of the $\mathrm{SiO} J=2 \rightarrow 1$ emission line as a function of that of the [OI] $63 \mu \mathrm{m}$ emission line for the four strongest shock features in the region. The $\mathrm{SiO}$ data have been smoothed to the $80^{\prime \prime}$ angular resolution of the ISO/LWS data for proper comparison.

rather than with an outflow. The relatively narrow lines would be in favor of this hypothesis. However millimetric and submillimetric continuum maps (Motte, private communication; Sandell, private communication) do not show any excess at that position. Dedicated high-resolution observations are necessary to disentangle the various velocity components in the area around W2 and to pin down the presence of this hypothetical source or outflow.

\subsection{Nature and age of the shocks}

Perhaps the most remarkable result of this study is the difference in the molecular/atomic emission of the different shocks present in the area. Because it can be up to 6 orders of magnitude more abundant in the shocks than in the quiescent ISM, SiO is an extremely useful probe of shocked material (Bachiller \& Perez Gutierrez 1997; Gueth et al. 1998; Lefloch et al. 1998). There is a general consensus that the large abundance enhancement seen in $\mathrm{SiO}$ results from the release into the gas phase of silicium previously trapped into the grains, either in their volatile mantles or in their refractory cores, followed by the oxidization of Si into SiO (Schilke et al. 1997; Caselli et al. 1997).

Atomic oxygen has often been argued to be also a good tracer of shocks, because strong $63 \mu \mathrm{m}$ emission is expected from the warm post-shocked gas found beyond dissociative shocks (Hollenbach 1985; Cohen et al. 1987; Ceccarelli et al. 1997). One would thus expect regions with strong $\mathrm{SiO}$ line emission to be associated with regions of strong [O I] $63 \mu \mathrm{m}$ emission. Instead, Fig. 8 shows that, not only do the strongest $\mathrm{SiO}$ peaks not correspond to the strongest [O I] $63 \mu \mathrm{m}$ emission peaks, but that there even seems to be an anti-correlation between the two! This is a priori unexpected because line emission from both $\mathrm{SiO}$ and [O I] surely originate, in the outflow.
This trend can be understood if the shocks traced by the $\mathrm{SiO}$ line emission are not dissociative, because in a non-dissociative shock, the atomic oxygen contained in the heated and compressed gas is efficiently incorporated into water molecules (e.g. Kaufman \& Neufeld 1996). This has been observed for example in HH54B by Liseau et al. (1996) or in HH7-11 by Molinari et al. (1999, 2000). Unfortunately our ISO observations were not sensitive enough to detect water emission in the outflow (see Sects. 2.2 and Sect. 3.4). This may be due to several reasons: a low filling factor, a low density (the $179 \mu \mathrm{m}$ line, the brightest one in HH54B and HH7-11, has a critical density much larger than $10^{5} \mathrm{~cm}^{-3}$ ) and finally a low $\mathrm{H}_{2} \mathrm{O}$ column density. Assuming a filling factor equal to unity, a density equal to $10^{5} \mathrm{~cm}^{-3}$, a temperature equal to $150 \mathrm{~K}$, as found in $\mathrm{E} 2$ (Sect. 4.2.1), and using the upper limit of $2 \times 10^{-12} \mathrm{erg} \mathrm{s}^{-1} \mathrm{~cm}^{-2}$ (Sect. 3.4), we derive an upper limit to the water column density of $N\left(\mathrm{H}_{2} \mathrm{O}\right) \leq 5 \times 10^{15} \mathrm{~cm}^{-2}$ (see Ceccarelli et al. 1998 for the details of the used LVG code). We emphasize that this is the $\mathrm{H}_{2} \mathrm{O}$ column density averaged on the $80^{\prime \prime}$ ISO beam. On the other hand, the $\mathrm{O}$ column density, derived from the [OI] $63 \mu \mathrm{m}$ line flux observed towards E2, is $6 \times 10^{15} \mathrm{~cm}^{-2}$. Therefore we found that in $\mathrm{E} 2$ the gaseous oxygen not locked into $\mathrm{CO}$ could be equally distributed between atomic oxygen and water. Note that these computations assume filling factors equal to unity, which is rather unlikely. Reducing the emitting region to $10^{\prime \prime}$ would decrease the $\mathrm{O} / \mathrm{H}_{2} \mathrm{O}$ ratio by about a factor two, making the water a more important gas coolant that the atomic oxygen. In conclusion, our $\mathrm{SiO}$ and [O I] $63 \mu \mathrm{m}$ observations suggest that the shocks around IRAS 16293-2422 are nondissociative, and therefore relatively slow $\left(\leq 40 \mathrm{~km} \mathrm{~s}^{-1}\right.$; Hollenbach 1998).

They are, however, sufficiently violent to release part of the dust grain content back into the gas phase, creating strong $\mathrm{SiO}$ and/or $\mathrm{H}_{2} \mathrm{CO}$ peaks. It is interesting that $\mathrm{E} 2$ is strong in $\mathrm{SiO}$ but weak in $\mathrm{H}_{2} \mathrm{CO}$, while the two other peaks (E1, W1, and perhaps W2) are seen in both species. Finally, HE1 and HE2 are strong in $\mathrm{H}_{2} \mathrm{CO}$, but weak in $\mathrm{SiO}$, probably because they lie on the edge the cloud. We argue that the different behavior of E2 when compared to E1 and W1 can be explained as a difference in the ages of the three shocks. Once injected into the gas phase, $\mathrm{SiO}$ and $\mathrm{H}_{2} \mathrm{CO}$ evolve differently. Chemical models predict that $\mathrm{H}_{2} \mathrm{CO}$ should disappear from the gas phase in $\sim 10^{4}$ yr (Charnley et al. 1992), whereas it takes about $10^{5}$ yr to transform $\mathrm{SiO}$ into $\mathrm{SiO}_{2}$ (Schilke et al. 1997). The simultaneous presence of $\mathrm{H}_{2} \mathrm{CO}$ and $\mathrm{SiO}$ emission in E1, therefore, points to a young shock, whereas E2 - only visible in $\mathrm{SiO}$ - could be older.

\section{Conclusions: The morphology and physics of the region}

The picture emerging from this study is sketched in Fig. 9. There are at least three sources in the area (16293A, $16293 \mathrm{~B}$ and $16293 \mathrm{E})$, possibly four, if W2 is a source 


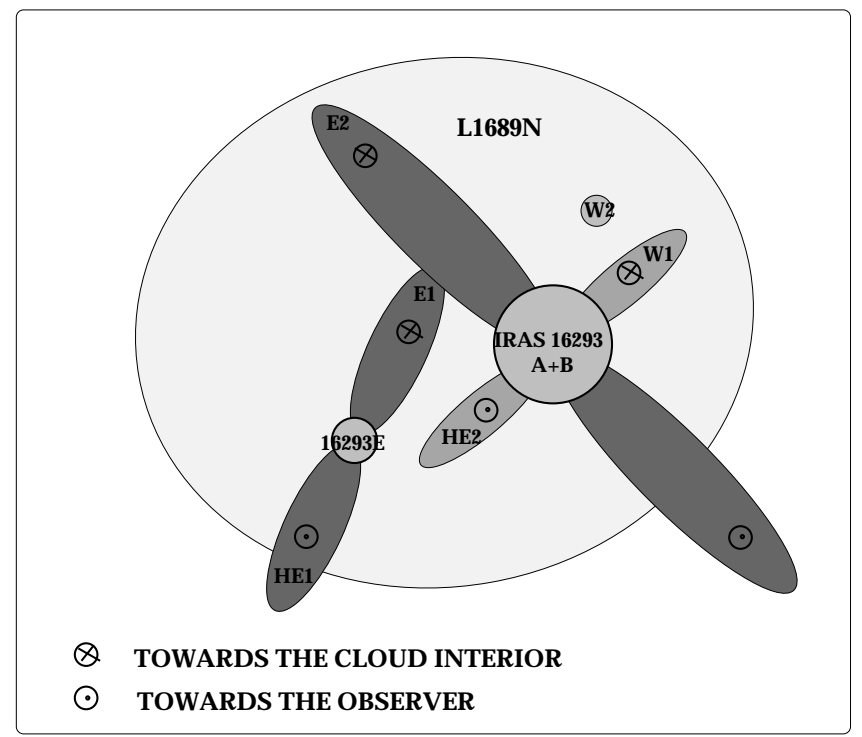

Fig. 9. Sketch of the region seen face on. The dark grey outflows have been clearly identified. Light gray used for the outflow emanating from IRAS 16293-2422 and the W2 source indicate that they are assumptions (see text). The noticeable asymmetry of the E1- HE1 and W1-HE2 outflows are not reproduced here.

(which is the hypothesis taken to draw the sketch). The host cloud, L1689N, seems to be more elongated in the direction perpendicular to the plane of the sky and the three well established sources (16293A, 16293B and 16293E) lie relatively close to the cloud edge towards us, whereas the hypothetical fourth source (W2) lies deeper inside the cloud.

IRAS 16293-2422, a well known Class 0 protostar, is surrounded by a massive envelope previously studied by several authors (e.g. van Dishoeck et al. 1995; Ceccarelli et al. 2000a,b and 2001). 16293E is a recently discovered Class 0 source (Loinard et al. in prep.) driving an outflow. Although its envelope is less massive than that of IRAS $16293-2422$, it is more prominent in the $\mathrm{N}_{2} \mathrm{H}^{+}$map, probably because it is colder. On the contrary, no conspicuous $\mathrm{H}_{2} \mathrm{CO}$ emission is seen associated with its envelope, probably because of the high $\mathrm{H}_{2} \mathrm{CO}$ emission of the cloud itself and the lower gas temperature.

There are at least two outflows in the region, perhaps three, or even four if W2 is a shocked region resulting from a masked outflow. In addition to the already known North-East - South-West outflow reported by Walker et al. (1986) we suggest that the protobinary IRAS 16293-2422 might power a second outflow oriented North-West - South-East. This suggestion rests only on the near alignment of shocked regions (W1 and HE2) located on both sides of the source. The third outflow emanates from 16293E. These three outflows are responsible for the appearance of four shocked regions (E1, E2, $\mathrm{W} 1$ and HE2) and maybe an additional one (HE1) where $\mathrm{SiO}, \mathrm{H}_{2} \mathrm{CO}$ and atomic oxygen emissions are enhanced. However, different molecular/atomic lines sometimes tend to give a different picture, i.e. seem to trace different shocks. The shocked regions which are well embedded in the cloud (E1, E2, W1 and W2) also show strong SiO emission. The presence of abundant $\mathrm{SiO}$ may be explained by the release of silicon enriched grain mantles inside the cloud with respect to $\mathrm{H}_{2} \mathrm{CO}$ or by partial destruction of the grains in the denser inner regions of the cloud.

The anticorrelation observed between the $\mathrm{SiO}$ and [O I] emission suggests that these shocks are non-dissociative, and consequently relatively slow $\left(\leq 40 \mathrm{~km} \mathrm{~s}^{-1}\right)$ and gentle. The simultaneous presence of $\mathrm{H}_{2} \mathrm{CO}$ and $\mathrm{SiO}$ emission towards E1 and W1 suggests that they are relatively young shocks ( $\left.\sim 10^{4} \mathrm{yr}\right)$, whereas E2, which only shows strong $\mathrm{SiO}$ emission, is a slightly older shock $\left(\sim 10^{5} \mathrm{yr}\right)$. Finally, in E1 and E2, two shocks at different velocities and with different densities are observed. We argued that they are the wind (the higher velocity component) and cloud (the lower velocity component) shocks respectively, foreseen by theory (e.g. Hollenbach 1998).

Acknowledgements. We thank the IRAM and SEST staff in Pico Veleta and La Silla for their assistance with the observations, and the IRAM and ESO Program Committee for their award of observing time. We also thank the LWS and SWS teams for their work on the ISO instruments. We are grateful to P. Caselli for helpful discussions on the shocks, and to our referee, P. Schilke, whose insightful and constructive comments helped to improve the quality of this paper.

\section{References}

André, P., Ward-Thompson, D., \& Barsony, M. 1993, ApJ, 406, 122

Bachiller, R., \& Pérez Gutiérrez, M. 1997, ApJL, 487, L93

Bachiller, R., \& Pérez Gutiérrez, M. 1998, in Herbig-Haro flows and the birth of low mass stars, ed. Bo Reipurth, \& C. Bertout, IAU Symp., 182, 153

Blake, G. A., van Dishoeck, E. F., Jansen, D. J., Groesbeck, T. D., \& Mundy, L. G. 1994, ApJ, 428, 680

Caselli, P., Myers, P. C., \& Thaddeus, P. 1995, ApJ, 455, L77

Caselli, P., Hartquist, T. W., \& Havnes, O. 1997, A\&A, 322, 296

Caux, E., Ceccarelli, C., Castets, A., et al. 1999, A\&A, 347, L1

Ceccarelli, C., Haas, M. R., Hollenbach, D. J., \& Rudolph, A. L. 1997, ApJ, 476, 771

Ceccarelli, C., Castets, A., Caux, E., et al. 2000a, A\&A, 355, 1129

Ceccarelli, C., Loinard, L., Castets, A., Tielens, A. G. G. M., \& Caux, E. 2000b, A\&A, 357, L9

Ceccarelli, C., Loinard, L., Castets, A., et al. 2001, A\&A, in press

Charnley, S. B., Tielens, A. G. G. M., \& Millar, T. J. 1992, ApJL, 399, L71

Clegg, P. E., Ade, P. A. R., Armand, C., et al. 1996, A\&A, 315, L38

Codella, C., \& Bachiller, R. 1999, A\&A, 350, 659

de Graauw, T., Haser, L. N., Beintema, D. A., et al. 1996, A\&A, 315, L49

Forveille, T., Guilloteau, S., \& Lucas, R. 1989, CLASS Manual (Grenoble: IRAM)

Gueth, F., Guilloteau, S., \& Bachiller, R. 1998, A\&A, 333, 287 
Hirano, N., Mikami, H., Umemoto, T., Yamamoto, S., \& Taniguchi, Y. 2000, ApJ, in press (HI2000)

Hollenbach, D. J. 1998, in Herbig-Haro flows and the birth of low mass stars, ed. Bo Reipurth, \& C. Bertout, IAU Symp., 182,181

Kaufman, M. J., \& Neufeld, D. A. 1996, ApJ, 456, 611

Kessler, M. F., Steinz, J. A., Anderegg, M. E., et al. 1996, A\&A, 315, L27

Knude, J., \& Hog, E. 1998, A\&A, 338, 897

Lefloch, B., Castets, A., Cernicharo, J., \& Loinard, L. 1998, ApJL, 504, L109

Liseau, R., Ceccarelli, C., Larsson, B., et al. 1996, A\&A, 315, 181

Loinard, L., Castets, A., Ceccarelli, C., et al. 2000, A\&A, 359, 1169

Loinard, L., Castets, A., Ceccarelli, C., Caux, E., \& Tielens, A. G. G. M. 2001, ApJL, in press

Looney, L. W., Mundy, L. G., \& Welch, W. J. 2000, ApJ, 529, 477

Martin-Pintado, J., Bachiller, R., \& Fuente, A. 1992, A\&A, 254,315
Mizuno, A., Fukui, Y., Iwata, T., Nozawa, S., \& Takano, T. 1990, ApJ, 356, 184

Molinari, S., Ceccarelli, C., White, G. J., et al. 1999, ApJL, 521, L71

Molinari, S., Noriega-Crespo, A., Ceccarelli, C., et al. 2000, ApJ, 538, 698

Schilke, P., Walmsley, C. M., Pineau des Forêts, G., \& Flower, D. R. 1997, A\&A, 321, 293

Swinyard, B. M., Clegg, P. E., Ade, P. A. R., et al. 1996, A\&A, 315, L43

Turner, B. E. 1995, ApJ, 449, 635

Turner, B. E., \& Thaddeus, P. 1977, ApJ, 211, 755

van Dishoeck, E. F., Blake, G. A., Jansen, D. J., \& Groesbeck, T. D. 1995, ApJ, 447, 760

van Dishoeck, E. F., \& Blake, G. A. 1998, ARA\&A, 36, 317

Walker, C. K., Lada, C. J., Young, E. T., Maloney, P. R., \& Wilking, B. A. 1986, ApJ, 309, L47

Womack, M., Ziurys, L. M., \& Wyckoff, S. 1992, ApJ, 387, 417

Ziurys, L. M., Friberg, P., \& Irvine, W. M. 1989, ApJ, 343, 301 